\title{
In-Situ Epoxidation of Castor Oil Using Heterogeneous Acidic Ion-Exchange Resin Catalyst (IR-120) for Bio-Lubricant Application
}

\author{
Venu Babu Borugadda and Vaibhav V. Goud* \\ Department of Chemical Engineering, Indian Institute of Technology-Guwahati \\ Guwahati, Assam 781039, India \\ *Corresponding author: vvgoud@iitg.ernet.in
}

( Manuscript received 16 June 2015; accepted 4 August 2015; published 15 November 2015 )

( Presented at ASIATRIB-2014, Agra, India, February 2014 )

\begin{abstract}
The present work reports the formulation of castor oil (CO) epoxide as a bio-lubricant basestock. The main objective of this study was to improve the physico-chemical properties and thermo-oxidative stability of CO via structural modification. Epoxidation reaction was carried out at $60^{\circ} \mathrm{C}$ for $10 \mathrm{~h}$ using heterogeneous acidic ion-exchange resin (IR-120) as a catalysts (15mass\%), acetic acid ( $0.5 \mathrm{~mol})$ as an oxygen carrier and hydrogen peroxide $(1.5 \mathrm{~mol})$ as an oxygen donor. Product was confirmed by ${ }^{1} \mathrm{H}-\mathrm{NMR}$, FTIR spectral analysis and epoxide content was determined by oxirane oxygen analysis. Further, significant physico-chemical properties such as viscosity, acid value (AV), pour point (PP) was determined for prepared $\mathrm{CO}$ epoxide. Thermo-oxidative stability of prepared epoxide and unmodified $\mathrm{CO}$ were compared by thermo gravimetric analysis (TGA) technique (in inert and oxygen atmospheres). Modified CO clearly illustrated significant improvement in the required physico-chemical properties and thermo-oxidative stability. Results of this study revealed that the prepared epoxide could be used as a potential bio-lubricant basestock in hydraulic lubricant application.
\end{abstract}

Keywords: Castor oil, Epoxidation, Oxirane oxygen content (OOC), Thermo gravimetric analysis, Ion-exchange resin (IER)

\section{Introduction}

A steady increase in the use of eco-friendly consumer products like lubricants has occurred as a result of gradual dwelling of world fossil reserves, strict government regulations and increased public awareness for a pollution-free environment. Currently, most widely used lubricant base oils include mineral oils, synthetic oils, re-refined and vegetable oils. Among these, mineral oils are the most commonly used; they consist predominantly of hydrocarbons, but also contain some sulphur and nitrogen compounds with traces of a number of metals. Due to their inherent toxicity and non-biodegradable nature, they pose a constant threat to ecology and vast ground water reserves [1-3].

Due to the negative impacts of mineral oils on ecosystem, lubricant industry has been trying to formulate renewable, bio-degradable lubricants with similar or even better in technical characteristics than those originated from mineral oils [1-3]. However, they are progressively being introduced into some specific applications, such as hydraulic fluids and lubricants for food industry. Among them, engine oils and hydraulic fluids are highly consumed and require special prerequisites like higher thermo-oxidative stability, improved cold flow properties and wide range of viscosity. During the search for alternatives, feed stocks with high content of oleic acid in the plant oils observed to be a potential alternative for biolubricant application $[4,5]$.

Vegetable oils are preferred, because not only they are biodegradable and non toxic, but also renewable in nature. Vegetable oils also show most of the specific properties required for lubricant applications such as high viscosity index, high lubricity, high flash point, very low volatility due to high molecular weight of the triacylglycerols. On the contrary, they possess lower thermal, oxidative, hydrolytic stabilities and poor low-temperature characteristics, which are mainly due to their fatty acid composition and presence of unsaturation. Conversion of unsaturation $(\mathrm{C}=\mathrm{C})$ to oxirane ring via epoxidation received special attention to synthesize bio-lubricant basestocks, because it opened up a wide range of feasible reactions that can be carried out under moderate reaction conditions due to 
high reactivity of oxirane ring [6,7]. Reported literature on synthesis of bio-lubricant revealed that, in most of the studies, edible oils has been used for biolubricant synthesis and very scanty information is available on the use of non edible oils. In this sense, the main objective of this work was to develop new environmental friendly biolubricant basestock with improved thermo-oxidative stability and better cold flow properties via structural modification of $\mathrm{CO}$ using IR-120 as a heterogeneous acidic catalyst.

\section{Materials and methods}

Mechanically extracted $\mathrm{CO}$ was procured from Cherukupalli (Andhra Pradesh, India). The respective fatty acid composition of $\mathrm{CO}$ is as follows: ricinoleic $84 \%$, linoleic $6 \%$, oleic $4 \%$, stearic $1.8 \%$, palamitic $1.4 \%$ and linolenic $0.6 \%$ [8]. Hydrogen peroxide (purity, $50 \% \mathrm{v} / \mathrm{v}$ ) was purchased from RANKEM, ion-exchange resin (Amberlite IR 120, strong acid), glacial acetic acid (purity, 99-100\%) was supplied by M/s Merck India Pvt. Ltd. All other chemicals and reagents like, ethanol, phenolphthalein indicator, hydrogen bromide, methyl violet indicator, sodium hydroxide pellets, potassium hydrogen phthalate were of analytical grade and purchased from M/s Merck India Pvt. Ltd and were used without further purification.

\subsection{Analytical characterisation methods}

Free fatty acids (FFA) content and AV of $\mathrm{CO}$ and its epoxide were estimated according to the American Oil Chemists' Society (AOCS) official method (Te 1a-64, 1997). Density and specific gravity were determined by using a specific gravity bottle. Similarly, experimental oxirane oxygen content (OOC) was detected by AOCS method and theoretical OOC was obtained by mathematical expression reported by Borugadda and Goud (a) [2]. ${ }^{1} \mathrm{H}-\mathrm{NMR}$ spectrum of CO and its epoxide were recorded using $600 \mathrm{MHz}$ NMR spectrometer. FTIR spectra was recorded on a Shimadzu Fourier transforms infrared spectroscopy (IR

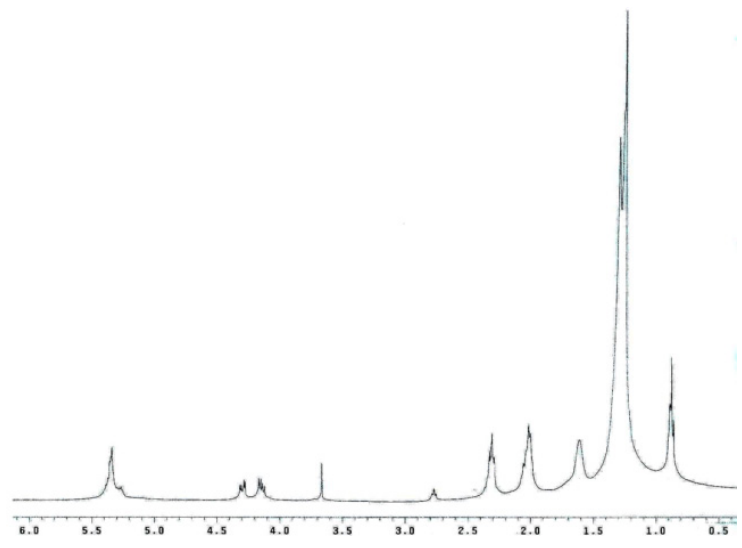

Fig. $1{ }^{1} \mathrm{H}-\mathrm{NMR}$ spectra of CO
Affinity 1, Shimadzu, Japan) equipped with a KBr beam splitter as described by Borugadda and Goud (b) [3]. Cold flow properties were determined by DSC (differential scanning calorimetry) using the procedure covered by Borugadda and Goud (c) [8], similarly TGA (thermo gravimetric analysis) was performed to estimate the thermal and oxidative stability as described previously [9].

\subsection{Synthesis of $\mathrm{CO}$ epoxide}

$\mathrm{CO}$ epoxidation reaction was carried out in isothermal conditions, using mechanical stirring (five bladed turbine type) in a cylindrical flat bottom three necked glass reactor $(750 \mathrm{ml})$. The entire setup was immersed in heating water bath. Following reaction conditions were adopted for $\mathrm{CO}$ epoxidation, $\mathrm{CO}$ unsaturation to hydrogen peroxide molar ratio $1: 1.5 ; 0.5$ moles acetic acid per mole of unsaturation and 15 mass $\%$ catalyst loading (IR-120). The reaction was continued for ten hours and during epoxidation 1000-1100 rpm stirring speed was maintained to ensure consistent mixing, under atmospheric pressure. Upon completion of epoxidation reaction and prior to analysis, epoxide was washed repeatedly with warm Millipore water $\left(40^{\circ} \mathrm{C}\right)$, washing was continued until all the catalyst and other unreacted reactants were removed to make it neutral. Solvent and water traces were removed using rotary evaporator. Final product was confirmed by ${ }^{1} \mathrm{H}-\mathrm{NMR}, \mathrm{FTIR}$ spectral techniques and OOC analysis. Significant physico-chemical properties of $\mathrm{CO}$ epoxide and $\mathrm{CO}$ were estimated and compared.

\section{Results and discussion}

\section{1. ${ }^{1} \mathrm{H}-\mathrm{NMR}$ spectroscopy}

Proton $\left({ }^{1} \mathrm{H}\right)$ NMR spectra has played an important role in studying the structure of $\mathrm{CO}$ and its epoxide product, as well as monitoring the epoxidation reaction. As shown in Figs. 1 and 2, the protons in $\mathrm{CO}$ and its epoxide display different chemical shifts in ${ }^{1} \mathrm{H}-\mathrm{NMR}$ spectra. The spectra at $\delta 5.3$ to $5.6 \mathrm{ppm}$ exhibit the

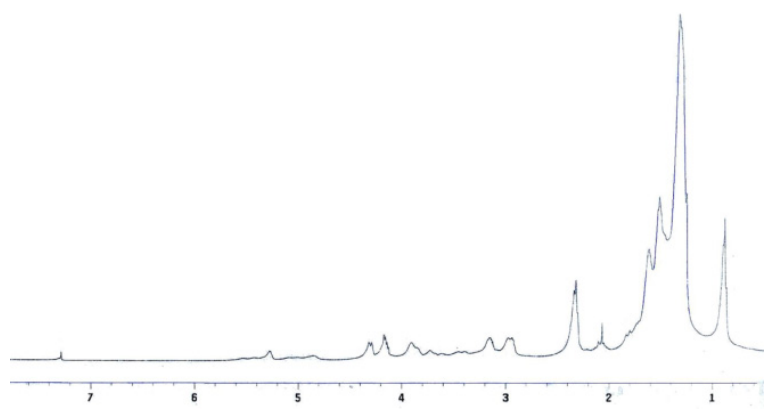

Fig. $2 \quad{ }^{1} \mathrm{H}-\mathrm{NMR}$ spectra of CO epoxide 


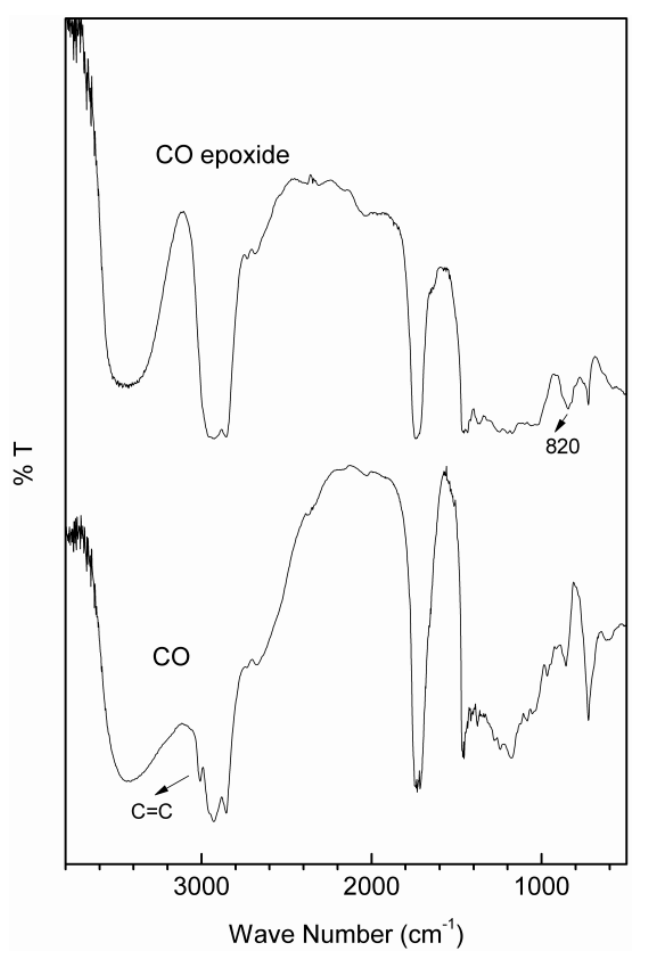

Fig. 3 FTIR spectrum of $\mathrm{CO}$ and its epoxide

presence of unsaturation in $\mathrm{CO}$ (Fig. 1), during the epoxidation all the unsaturation content was transformed into oxirane ring (Fig. 2). Epoxide product was confirmed by the disappearance of unsaturation content in CO spectra at $\delta 5.3$ to $5.6 \mathrm{ppm}$ (Fig. 1) and the appearance of new chemical shifts at $\delta 2.9-3.2 \mathrm{ppm}$ (Fig. 2). Alike observations were noticed by Borugadda and Goud (b) [3] during their study on epoxidation of waste cooking oil.

\subsection{FTIR spectral analysis}

The FTIR spectra of $\mathrm{CO}$ and its epoxide are shown in Fig. 3. The FTIR spectra clearly indicates the disappearance of unsaturated double bonds in $\mathrm{CO}$ at $3010 \mathrm{~cm}^{-1}$ (Fig. 3), similarly from the spectra of $\mathrm{CO}$ epoxide, disappearance of double bonds at $3010 \mathrm{~cm}^{-1}$ was observed and appearance of peaks at $815-840 \mathrm{~cm}^{-1}$ (Fig. 3) was considerably intensified, signifying that all the unsaturation was turned into OOC (epoxy groups). In the current study, $\mathrm{CO}$ epoxide functional group was detected from $820 \mathrm{~cm}^{-1}$, but in general epoxy groups start detecting at $823 \mathrm{~cm}^{-1}$ : this may be due to the shift in the peak. Borugadda and Goud (b) [3] reported that the presence of epoxy groups at $823-843 \mathrm{~cm}^{-1}$, which agrees well with this study.

\subsection{Low temperature mobility of $\mathrm{CO}$ and its epoxide}

The fluidity of $\mathrm{CO}$ epoxide below the ambient temperature is determined by its pour point (PP), defined as the temperature at which complete crystallization of the epoxide could be observed. In the

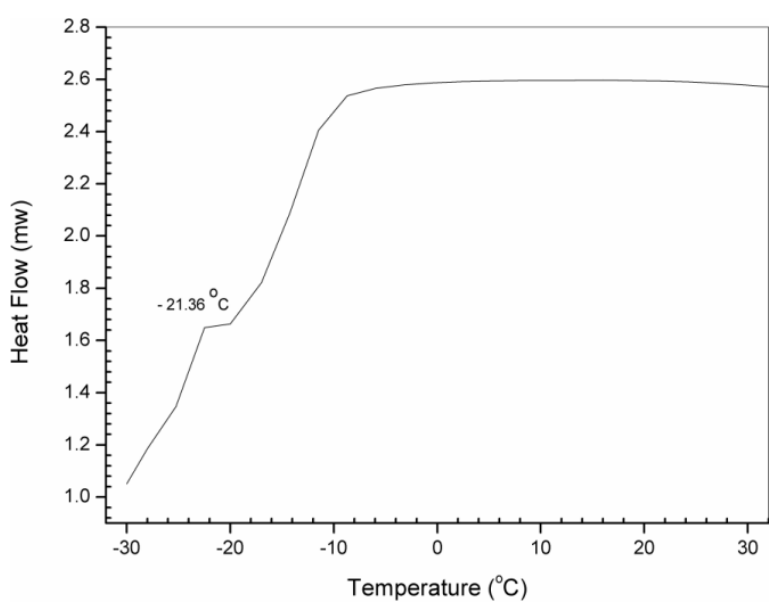

Fig. 4 DSC thermogram for CO

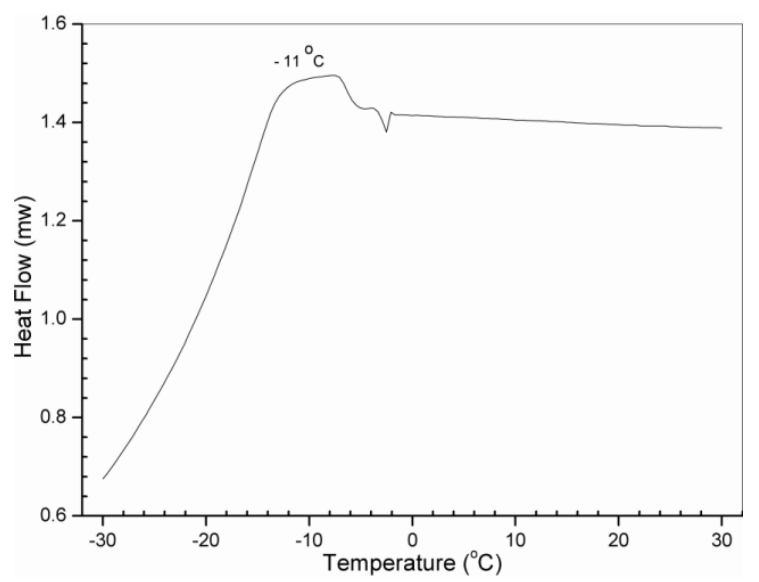

Fig. 5 DSC thermogram for CO epoxide

current study, PP was determined by DSC thermal analysis technique, $\mathrm{CO}$ and its epoxide thermograms were presented in Figs. 4 and 5. The PP's of $\mathrm{CO}$ and its epoxide were noticed as a sharp increase in heat flow due to exothermic nature of increasing reaction. The point at which exothermic peak occurs was considered as pour point temperature. From CO DSC thermo gram (Fig. 4), PP was found to be $-21.36^{\circ} \mathrm{C}$ which was quite close to earlier reported value (Borugadda and Goud) [9], similarly for $\mathrm{CO}$ epoxide it was found to be $-11^{\circ} \mathrm{C}$ (Fig. 5). After structural modification (i.e. epoxidation) variation in the $\mathrm{PP}$ of $\mathrm{CO}$ and its epoxide were well explained by Borugadda and Goud (b) [3]. Therefore, from the present study, it can be concluded that prepared epoxide can be potentially utilized below the ambient temperature up to $-11^{\circ} \mathrm{C}$, further to improve the $\mathrm{PP}$ oxirane ring opening of epoxides can be carried out as reported by Kamalakar et al. [10].

3.4. Thermo-oxidative stability of $\mathrm{CO}$ and its epoxide

Thermo-oxidative stability thermograms of $\mathrm{CO}$ and its epoxide are presented in Fig. 6, stability in the inert and oxidative atmospheres was determined by onset and 

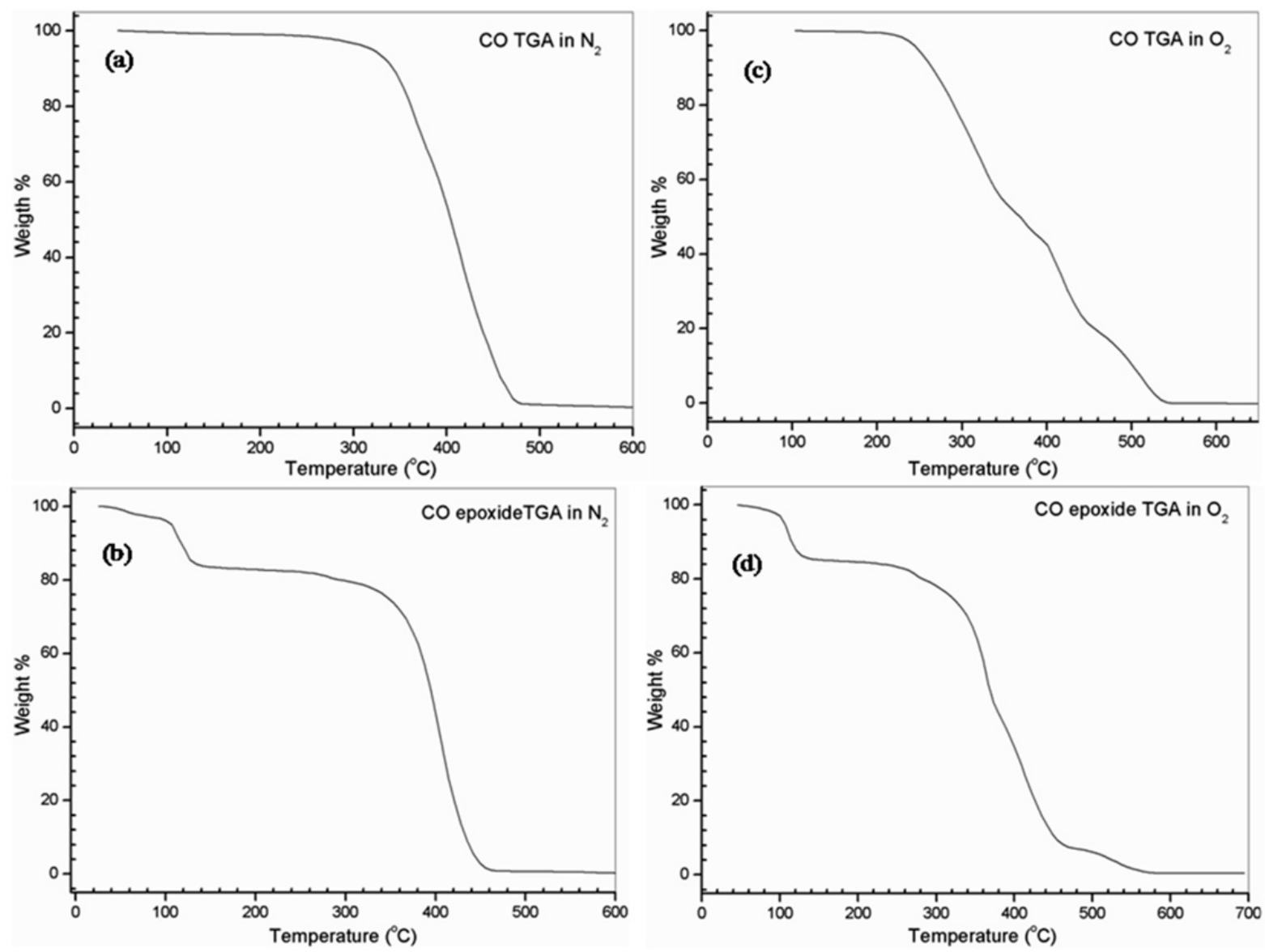

Fig. 6 TGA thermograms for CO (a), CO epoxide (b) in inert atmosphere, CO (c), CO epoxide (d) in oxygen atmosphere

oxidative onset temperatures. Onset temperature corresponds to the minimum temperature at which sample decomposition starts; in other words, maximum temperature up to which sample remains stable without any weight loss. From Fig. 6, it can be seen that thermal stability (onset temperature) of structurally modified $\mathrm{CO}$ (epoxide) was found to be $362^{\circ} \mathrm{C}$, which was improved significantly after epoxidation compared to unmodified $\mathrm{CO}\left(320^{\circ} \mathrm{C}\right)$. Likewise, from Fig. 6 oxidative stability (oxidative onset temperature) of $\mathrm{CO}$ epoxide was found to be $320^{\circ} \mathrm{C}$, which was higher than the $\mathrm{CO}$ epoxide $\left(240^{\circ} \mathrm{C}\right)$. Comparing the thermal and oxidative stability, oxidative stability of epoxide was improved by $80^{\circ} \mathrm{C}$. This difference in the onset and oxidative onset temperatures can be justified with the help of structural modification of $\mathrm{CO}$. As reported by Borugadda and Goud [9], unsaturation content $(\mathrm{C}=\mathrm{C}$, $86.57 \mathrm{gI}_{2} / 100 \mathrm{~g}$ of oil) in triglyceride structure hinders the thermo-oxidative stability, whereas after structural modification unsaturation in $\mathrm{CO}$ epoxide reduced to $6.34 \mathrm{gI}_{2} / 100 \mathrm{~g}$ of epoxide. Therefore, thermal and oxidative stability of $\mathrm{CO}$ epoxide improved significantly, so that the prepared $\mathrm{CO}$ epoxide can be used potentially up to $362^{\circ} \mathrm{C}$ and $320^{\circ} \mathrm{C}$ in inert and oxidative atmospheres respectively. However, in both the thermograms (Figs. 6 (b) and (d)) step like trend was found between 100 and $140^{\circ} \mathrm{C}$; this could be attributed to moisture content in the epoxide. In general, moisture evaporates at around $90-100^{\circ} \mathrm{C}$, since the moisture is bounded with epoxide, evaporation of bounded moisture consumed more heat energy to evaporate i.e., up to $140^{\circ} \mathrm{C}$.

\subsection{Physico-chemical characteristics of castor oil and its epoxide}

Epoxidized plant seed oils can be used as lubricant base fluids due to their viscosity and higher thermo-oxidative stability compared to structurally unmodified oils. Physico-chemical properties of $\mathrm{CO}$ (extracted by mechanical extraction) and its epoxide are shown in Table 1. The AVs of CO and its epoxide were found to be 26.74 and $1.32 \mathrm{mgKOH} / \mathrm{g}$ respectively. During the study, density was found to be improved for epoxidised CO $\left(886.49 \mathrm{~kg} / \mathrm{m}^{3}\right)$ than raw CO $(819.23$ $\mathrm{kg} / \mathrm{m}^{3}$ ), similarly kinematic viscosity $\left(\right.$ at $40^{\circ} \mathrm{C}$ ) for $\mathrm{CO}$ and its epoxide was found to be 232.64 and 286.83 $\mathrm{mm}^{2} / \mathrm{s}$ respectively. This increase in viscosity may be due to addition of the oxygen molecules in the midst of unsaturation (double bond), thereby the intermolecular forces, molecular weight and polarity increase. From 
Table 1 Physico-chemical properties of CO, $\mathrm{CO}$ epoxide and their characterization methods

\begin{tabular}{lccc}
\hline Properties & CO & CO Epoxide & Standard Method \\
\hline Acid Value $(\mathrm{mgKOH} / \mathrm{g})$ & 26.74 & 1.32 & AOCS (Te 1a-64, 1997) \\
Density $\left(\mathrm{kg} / \mathrm{m}^{3}\right)$ & 819.23 & 886.49 & ASTM D 4052-91 \\
Iodine Value $\left(\mathrm{IV}, \mathrm{gI}_{2} / 100 \mathrm{~g}\right.$ of epoxide) & 86.57 & 6.34 & AOCS (Tg 1-64, 1997) \\
Kinematic Viscosity $\left(\mathrm{mm}^{2} / \mathrm{s}\right)$ at $40{ }^{\circ} \mathrm{C}$ & 232.64 & 286.83 & ASTM D-445 \\
Oxirane Content (Experimental, mass \%) & - & 4.84 & AOCS Cd-9, 120 \\
Oxirane Content (Theoretical, mass \%) & - & 5.05 & - \\
Relative percentage & - & 95.84 & - \\
Conversion of oxirane $(\%)$ & & & \\
\hline
\end{tabular}

this study, it was also observed that the epoxidized $\mathrm{CO}$ has higher molecular weight and more polar structure than unmodified $\mathrm{CO}$; consequently, the interactions between molecules would be stronger. Stronger interactions among the molecules can form as a film between the moving surfaces, therefore, reduces friction between the rubbing surfaces; this property of $\mathrm{CO}$ epoxide enhances the lubricity in a dynamic system. Likewise, theoretical and experimental oxirane content were found to be 5.05 and 4.84 mass\% respectively. From the relative percentage conversion of oxirane it could be claimed that $95.8 \%$ of double bonds were converted to oxirane oxygen, similar observation can be made and justified from iodine value as well. All these physico-chemical properties have a great significance on the performance of final lubricant basestock and shows that the prepared lubricant can be used as a replacement for conventional hydraulic lubricant basestocks.

\section{Conclusions}

This work represents the first comprehensive report on synthesis and maximization of castor oil epoxide from raw $\mathrm{CO}$ containing moderate free fatty acids. Product was confirmed by ${ }^{1} \mathrm{H}-\mathrm{NMR}$, FTIR, IV and oxirane analysis techniques and all the significant physico-chemical properties were determined for the final epoxide product and compared with the unmodified product. From these investigations, it was observed that physico-chemical properties were improved significantly, especially thermo-oxidative stability of the prepared epoxide detected satisfied stability. Hence, the prepared CO epoxide can be used as an in-exhaustible, eco-friendly alternative for the conventional lubricant basestock.

\section{References}

[1] Campanella, A., Fontanini, C. and Baltanas, M. A., "High Yield Epoxidation of Fatty Acid Methyl Esters with Performic Acid Generated In-Situ," Chemical Engineering Journal, 144, 3, 2008, 466-475.

[2] Borugadda, V. B. and Goud, V. V., "Epoxidation of Castor Oil Fatty Acid Methyl Esters (COFAME) as a Lubricant Base Stock Using Heterogeneous Ion-Exchange Resin (IR-120) as a Catalyst," Energy Procedia, 54, 2014 (a), 75-84.

[3] Borugadda, V. B. and Goud, V. V., "Synthesis of Waste Cooking Oil Epoxide as a Bio-Lubricant Basestock: Characterization and Optimization Study," Journal of Bioprocess Engineering and Biorefinery, 3, 2014 (b), 57-72.

[4] Erhan, S. Z., Sharma, B. K., Liu, Z. and Adhvaryu, A., "Lubricant Base Stock Potential of Chemically Modified Vegetable Oils," Journal of Agricultural and Food Chemistry, 56, 2008, 8919-8925.

[5] Hwang, H, S. and Erhan, S. Z., "Synthetic Lubricant Basestocks from Epoxidized Soybean Oil and Guerbet Alcohols," Industrial Crops and Products, 23, 2006, 311-317.

[6] Lathi, P. S. and Mattiasson, B., "Green Approach for the Preparation of Biodegradable Lubricant Base Stock from Epoxidised Vegetable Oil," Applied Catalysis B: Environmental, 69, 2007, 207-212.

[7] Salimon, J. and Salih, N., "Chemical Modification of Oleic Acid for Biolubricant Industrial Applications," Australian Journal of Basic and Applied Sciences, 4, 7, 2010, 1999-2003.

[8] Borugadda, V. B. and Goud, V. V., "Thermal, Oxidative and Low Temperature Properties of 
Methyl Esters Prepared from Oils of Different Fatty Acids Composition: A Comparative Study," Thermochemica Acta, 577, 2014 (c), 33-40.

[9] Borugadda, V. B. and Goud, V. V., "Comparative Studies of Thermal, Oxidative and Low Temperature Properties of Waste Cooking Oil and
Castor Oil," Journal of Renewable and Sustainable Energy, 5, 2013, 063104.

[10] Kamalakar, K., Rajat, A. K., Prasad, R. B. N. and Karuna, M. S. L., "Rubber Seed Oil-Based Biolubricant Base Stocks: A Potential Source for Hydraulic Oils," Industrial Crops and Products, 51, 2013, 249-257. 